\title{
A novel ferroptosis-related 12-gene signature predicts clinical prognosis and reveals immune relevancy in clear cell renal cell carcinoma
}

\section{Yingkai Hong}

The First Affiliated Hospital of Shantou University Medical College

Mingen Lin

The First Affiliated Hospital of Shantou University Medical College

Dehua Ou

The First Affiliated Hospital of Shantou University Medical College

\section{Zhuangkai Huang}

The First Affiliated Hospital of Shantou University Medical College

Peilin Shen ( $\nabla$ plshen@stu.edu.cn )

The First Affiliated Hospital of Shantou University Medical College https://orcid.org/0000-0003-35843449

Primary research

Keywords: ferroptosis, gene signature, clear cell renal cell carcinoma, bioinformatics

Posted Date: January 29th, 2021

DOl: https://doi.org/10.21203/rs.3.rs-153932/v1

License: (c) (i) This work is licensed under a Creative Commons Attribution 4.0 International License. Read Full License 


\section{Abstract}

Background Clear cell renal cell carcinoma (ccRCC) is still highly aggressive and lethal even with various therapeutic approaches. As kidney is an iron-metabolism-related organ, exploring and assessing the clinical value of ferroptosis, an iron-dependent regulated cell death, is practical and significant. Methods Prognostic ferroptosis-related differentially expressed genes (DEGs) were identified from KIRC cohort in TCGA database, from which a prognostic signature was established using the Lasso-penalized Cox regression analysis. Each patient in the KIRC cohort and the E-MTAB-1980 cohort (from the ArrayExpress database) was assigned with a calculated signature-correlated risk score and categorized to be either in high-or low-risk group divided by the median risk score in the KIRC cohort. Then, the independent prognostic value of the signature was further assessed by Kaplan-Meier (K-M) survival, time-dependent receiver operating characteristic (ROC) and Cox regression analyses base on overall survival (OS) in both cohorts. Lastly, risk-related DEGs were identified in both cohorts and applied with the enrichment analyses for Gene Ontology (GO), Kyoto Encyclopedia of Genes and Genomes (KEGG) and immune infiltration. Results Within 60 ferroptosis-related genes, 32 prognostic DEGs were identified, from which we constructed a prognostic 12-gene signature including CARS1, HMGCR, CHAC1, GOT1, CD44, STEAP3, AKR1C1, CBS, DPP4, FANCD2, SLC1A5 and NCOA4. Patients in both cohorts were divided into high- and low-risk group which were visually distributed in two sets and with positive-risk-related mortality. The K-M survival and the ROC curves validated the signature as prognostic valuable with $\mathrm{P}<0.05$ and area under the curve $>0.7$ in both cohorts, respectively. Multivariate Cox regression further confirmed the risk score as an independent prognostic predictor for OS. Commonly enriched term in GO and KEGG not only shown a highly iron correlation, but also, interesting, an immunity relevancy of 3 immune cells (macrophages, mast cells and regulatory $\mathrm{T}$ cell) and 1 immune-related function (antigen processing cell co-stimulation). Conclusion We established a novel 12 ferroptosis-related-gene signature which was proved as an independent prognostic predictor for OS and inferred as relating to tumor immunity in CCRCC, however, the underlying mechanism is still poorly characterized and needed further exploration.

\section{Background}

As the latest research suggested, renal cell carcinoma (RCC) is the second common diagnostic urological cancer after bladder cancer, in which about $80 \%$ are clear cell RCC (ccRCC) $(1,2)$. Even with various therapeutic approaches like surgery, chemotherapy, radiotherapy, targeted therapy and the newly proposed immunotherapy, ccRCC is still one of the toughest clinical problems in urology. Delay in diagnosis and high metastatic rate bear the main responsibility. The incidence of advanced ccRCC is about $33 \%$ at patients' first hospital visits, in which $40 \%$ develop distant metastases and suffered from poor survival outcomes (5-year survival rate is less than $11.2 \%$ ) (3). For localized RCC, radical nephrectomy is still the priority treatment modality. While concerning metastatic state tumors, conventional therapeutic methods like multitarget tyrosine kinase inhibitors (TKIs) and mammalian target of rapamycin (mTOR) inhibitors are extensively adopted, however, the therapeutic benefits are only modest (4). 
Iron is an essential element in the basic biological processes of human body, and the metabolism disorder of which is involved with the occurrence and progression of many tumors $(5,6)$. Recently, an iron-dependent regulated cell death (RCD) namely ferroptosis has been drawing more and more attentions in the cellular-molecular field of tumor. Less than a decade ago, ferroptosis was introduced as a non-apoptotic RCD distinguished from necroptosis (7), pyroptosis (8), and alkaliptosis $(9,10)$. In 2012, Dixon et al. (11) first demonstrated that instead of apoptotic inhibitors, the growth inhibition effect of erastin for RAS-mutant cancer cells can be completely antagonized by iron chelators and lipophilic antioxidants relying on a new form of RCD named ferroptosis. Morphologically, unlike typical apoptotic features such as membrane blebbing and shrinkage, classical necrosis-like features like cell swelling and plasma membrane rupture can be observed during ferroptosis (12). Biochemically, ferroptosis is driven by the reactive oxygen species (ROS) which was highly associated with iron accumulation and lipid peroxidation (13). Due to the high metabolic characteristic, most tumors are in a state of high oxidative stress and required to increase the ROS scavenging ability to prevent oxidative damage, which may make them sensitive to ferroptosis (14). Many cancers have been proved as ferroptosis-related, such as hepatocellular carcinoma (15), gastric cancer $(16,17)$, ovarian cancer $(18,19)$, and breast cancer $(20,21)$. Therefore, inducing ferroptosis to promote cell death or inhibit cell growth for cancer could be a new therapeutic potential (22).

As we know, kidney is an iron-metabolism-related organ with bio-functions like balancing iron homeostasis by filtering and reabsorbing iron as well as promoting hemoglobin synthesis by forming erythropoietin (23). Several studies have demonstrated that ccRCC is highly associated with iron metabolism $(24,25)$. Yet the role of ferroptosis in ccRCC remains poorly understood. Thus, exploring the potential correlation between ccRCC and ferroptosis is practical and significant.

To explore and assess the clinical value of ferroptosis in ccRCC, we performed this bioinformatics analysis by establishing an independently prognostic ferroptosis-related-gene signature using the TCGA database and validated it in the ArrayExpress database. Then, common functional annotations in both cohorts were screened out with Gene Ontology (GO), Kyoto Encyclopedia of Genes and Genomes (KEGG) and immune infiltration enrichment analyses to explore the underlying mechanisms.

\section{Methods}

The flow chart of the bioinformatics analysis is presented in Fig. 1. All the statistical analyses were completed in R language software (Version 4.0.3) (26), and a $\mathrm{P}<0.05$ was considered as statistically significant without a specified setting.

\subsection{Acquisition of ferroptosis-related genes and ccRCC cohorts}

A comprehensive literature survey about ferroptosis was performed and the high-quality articles were retrieved, in which 60 ferroptosis-related genes were fetched and presented in Supplementary Table S1 
$(27-30)$.

The derivation set and validation set of ccRCC were retrieved from the KIRC cohort (including $526 \mathrm{ccRCC}$ tissue samples and 72 normal kidney tissue samples) in the TCGA database (https://portal.gdc.cancer.gov/repository) and accession E-MTAB-1980 (including 101 ccRCC tissue samples) in the ArrayExpress database (www.https://www.ebi.ac.uk/arrayexpress), respectively. Both raw count values of gene expression and clinical information were download from the corresponding databases. The gene expression profiles of the KIRC and the E-MTAB-1980 cohort were normalized with the "edgeR" package (31) in R language software. Patients with 0 follow-up day would be removed from further analysis. Since all retrieved data were from public databases, no ethical review and approval from an Ethics Committee was required.

\subsection{Identifying differentially expressed ferroptosis-related genes with prognostic values between ccRCC tissues and normal kidney tissues}

The gene expression profiles of the KIRC cohort were applied with differential expression analysis using the "edgeR" R package. Differentially expressed genes (DEGs) between ccRCC tissues and normal kidney tissues were screened out with a false discovery rate $(F D R)<0.05$ and |fold change $(F C) \mid>1$. DEGs related to ferroptosis were selected and demonstrated with a heat map generated by the "pheatmap" $R$ package (32). Then, the univariate Cox analysis of overall survival (OS) for 60 ferroptosis-related genes was performed to identify the prognostic valuable ones. A Cox P-value $<0.05$ was considered significantly relating to OS. Ferroptosis-related DEGs with prognostic values were picked out using intersection analysis of DEGs and prognostic valuable genes with the "venn" R package (33) and then visualized with a protein-protein interaction (PPI) network generated by the STRING database (version 11.0) (34) and a correlation network generated by the "igraph" (35) and the "reshape2" (36) R packages.

\subsection{Construction and validation of a prognostic ferroptosis- related gene signature}

To minimize the risk of overfitting, we used the Lasso-penalized Cox regression analysis to rule out genes with overfitting tendency and construct a prognostic signature with the "glmnet" R package (37-39). The risk scores in the derivation set and the validation set were calculated according to a linear combination of the normalized expression value of each prognostic ferroptosis-related DEGs and its corresponding multivariate Cox regression coefficient $(\beta)$. The risk score calculation formula was as follow: Risk score= $(\beta \times$ expression value of CARS1 $)+(\beta \times$ expression value of HMGCR $)+(\beta \times$ expression value of CHAC1 $)+$ $(\beta \times$ expression value of GOT1 $)+(\beta \times$ expression value of CD44) $+(\beta \times$ expression value of STEAP3 $)+(\beta$ $\times$ expression value of AKR1C1 $)+(\beta \times$ expression value of CBS $)+(\beta \times$ expression value of DPP4 $)+(\beta \times$ expression value of FANCD2 $)+(\beta \times$ expression value of SLC1A5) $+(\beta \times$ expression value of NCOA4). In both sets, each patient was given a risk score from the calculation of the formula and then assigned to either high- or low-risk group divided by the median risk score of the derivation set. The distribution 
patterns were described for the risk scores and the corresponding survival times of all patients with scatter diagrams by the "pheatmap" R package, the gene expression of established signature with principal component analysis (PCA) by the "stats" $\mathrm{R}$ package (26), and the patients in different risk groups with t-distributed stochastic neighbor embedding (t-SNE) by the "Rtsne" R package (40). KaplanMeier (K-M) survival analysis and time-dependent receiver operating characteristic (ROC) analysis based on OS were performed using the "survival" package (41), the "survminer" package [42] and the "timeROC" package [43] in $\mathrm{R}$ to estimate the prognostic accuracy of the gene signature in the derivation set and verify it in the validation set.

\subsection{Prognostic independence of the gene signature from traditional clinical characteristics}

To further assess the independent prognostic value of the established gene signature, we used the univariate and multivariate Cox regression analyses to determine whether it was affected by other clinical characteristics. Several available clinical characteristics including age, gender and TNM stage were transformed into dichotomous variables and included for the calculation of hazard ratios (HRs) and 95\% confidence intervals (Cls) based on OS. P $<0.05$ was considered as statistically significant.

\subsection{GO, KEGG and immune infiltration enrichment analyses for risk-related DEGs}

According to the risk grouping, normalized gene expression matrixes of the derivation set and the validation set generated above were applied with "limma" R package (42) to identify risk-related DEGs with the cut-off criteria of $|\mathrm{FC}| \geq 1.5$ and FDR $<0.05$, respectively. Risk-related DEGs were analyzed with GO (43) and KEGG (44) using the "clusterProfiler" R package (45). The top 30 enriched terms in 3 categories of GO (including biological process (BP), cellular component (CC) and molecular function (MF)) and KEGG with the cut-off criteria of gene count $>10$ and P-value $<0.05$ in both 2 sets were intersected to obtain the overlapping enriched terms. Then, single-sample gene set enrichment analysis (ssGSEA) (46) for immune infiltration was applied with the "GSVA" R package (47) to assess the infiltrating score of 16 immune cells and the activity of 13 immune-related functions. With the annotated gene sets provided in Supplementary Table S2, we quantified the immune infiltration enrichment scores for different immune cells and immune-related functions to further investigate the correlation between risk score and immune status.

\section{Results}

\subsection{Identification of prognostic ferroptosis-related DEGs in the KIRC cohort}

Within 60 ferroptosis-related genes, 55 (91.67\%) were expressed differentially between ccRCC samples and normal kidney samples (Fig. 2a) and 34 (56.67\%) were considered as OS-related in the univariate Cox 
regression analysis (Fig. 2b), from which 32 overlapping genes correlated to ferroptosis and OS were picked out by the intersection analysis. Interactions of 32 prognostic ferroptosis-related DEGs were further visualized with the PPI and the correlation networks (Fig. 3a-b).

\subsection{Establishment of a ferroptosis-related 12-gene signature in the KIRC cohort}

The 32 prognostic ferroptosis-related DEGs were applied with the Lasso Cox regression analysis based on OS and a 12-gene signature including CARS1, HMGCR, CHAC1, GOT1, CD44, STEAP3, AKR1C1, CBS, DPP4, FANCD2, SLC1A5 and NCOA4 was identified in the KIRC cohort. According to the median risk score, patients were divided into high-risk group $(n=263)$ and low-risk group $(n=263)($ Fig. 4a), which were distributed in two sets in PCA and t-SNE (Fig. 4b-c). Besides, considering survival outcomes, we observed that the high-risk group was with more deaths than the low-risk group (Fig. 4d). To further evaluated the prognostic value and predictive performance of the gene signature, K-M survival and time-dependent ROC analyses were carried out and both come up with significant results. The K-M survival curve showed significantly worse survival outcomes for patients in the high-risk group compare to patients in the lowrisk group $(P=3.83 e-14)$ (Fig. 4e), and the area under the curve (AUC) reached 0.761 at 1 year, 0.735 at 3 years, 0.765 at 5 years, and 0.825 at 10 years in the ROC analysis (Fig. 4f).

\subsection{Validation of the prognostic predictive performance for the 12-gene signature in the E-MTAB-1980 cohort}

By applying the KIRC median risk score in the E-MTAB-1980 cohort, 101 patients were categorized as either high-risk $(n=53)$ or low-risk $(n=48)$ with different PCA, t-SNE and death probability distribution similar to the KIRC cohort (Fig. 5a-d). Consistently, a positive risk-related K-M survival curve with a significant P-value (2.514e-2) and ROC curves with considerable AUCs ( 0.733 at 1 year, 0.774 at 3 years, 0.763 at 5 years, and 0.721 at 10 years) were also established as convincing validation for the gene signature (Fig. 5e-f).

\subsection{Prognostic independence of the 12-gene signature from clinical characteristics}

Age, gender and TNM stage were included to test the independence of the prognostic 12-gene signature in the univariate and multivariate Cox regression. As shown in the univariate Cox regression analysis, risk score and TNM stage were proved as highly OS-related factors in both the KIRC (risk score: HR $=3.950$, $95 \% \mathrm{Cl}=3.031-5.147, \mathrm{P}<0.001$; TNM stage: $\mathrm{HR}=3.961,95 \% \mathrm{Cl}=2.871-5.463, \mathrm{P}<0.001)$ and the $\mathrm{E}-$ MTAB-1980 cohort (risk score: $\mathrm{HR}=10.247,95 \% \mathrm{Cl}=3.604-29.136, \mathrm{P}<0.001$; TNM stage: $\mathrm{HR}=7.472$, $95 \% \mathrm{Cl}=3.191-17.496, \mathrm{P}<0.001)$, as well as age in the $\mathrm{KIRC}$ cohort $(\mathrm{HR}=1.628,95 \% \mathrm{Cl}=1.202-2.204$, $\mathrm{P}=0.002$ ) (Fig. 6). After independently corrected by other clinical characteristics in the multivariate Cox regression, risk score was still a solid prognostic predictor for OS in both cohorts (KIRC: HR $=2.953,95 \%$ $\mathrm{Cl}=2.223-3.924, \mathrm{P}<0.001 ; \mathrm{E}-\mathrm{MTAB}-1980: \mathrm{HR}=4.270,95 \% \mathrm{Cl}=1.465-12.439, \mathrm{P}=0.008)$ (Fig. 7). 


\subsection{GO and KEGG enrichment analyses in the KIRC and the E-MTAB-1980 cohort}

After differential expression analysis between high- and low-risk group, 8597 DEGs significantly enriched in $958 \mathrm{BP}, 92 \mathrm{CC}, 155 \mathrm{MF}$ and $66 \mathrm{KEGG}$ terms were identified in the KIRC cohort. As for the E-MTAB-1980 cohort, 1253 DEGs were significantly enriched in 605 BP, 50 CC, 76 MF and 28 KEGG terms. All significantly enriched terms are shown in Supplementary Table S3 and S4 for the KIRC and the E-MTAB1980 cohort, respectively. We selected the top 30 enriched terms in GO and KEGG in both cohorts and found 9, 15, 20 and 8 overlapping enriched terms in BP, CC, MF and KEGG, respectively (Fig. 8-9). As expected, several iron-related molecular functions including metal ion transmembrane transporter activity, ion channel activity, and active ion transmembrane transporter activity were spotted. Moreover, what draws our attentions is that several immune-related terms in BP (humoral immune response), MF (cytokine receptor binding; cytokine activity) and KEGG (viral protein interaction with cytokine and cytokine receptor; IL-17 signaling pathway; cytokine-cytokine receptor interaction) were significant enriched in both cohorts (Fig. 8-9).

\subsection{Immune infiltration ssGSEA in the KIRC and the E-MTAB- 1980 cohort}

In the KIRC cohort, we found that 9 out of 16 immune cells were with significantly higher infiltration enrichment scores in the high-risk group including CD8 + T cells, macrophages, plasmacytoid dendritic cells ( $\mathrm{pDCs}$ ), T helper cells, follicular helper T cell (Tfh), helper T cells 1 (Th1 cells), helper T cells 2 (Th2 cells), tumor infiltrating lymphocyte (TIL) and regulatory T cell (Treg), while immature dendritic cells (iDCs) and mast cells were just the opposite (Fig. 10a). Similarly, high-risk group was predicted to be significantly correlated to most immune-related functions, except for Type II immune interferon (IFN) Response (Fig. 10b). Regarding SSGSEA in the E-MTAB-1980 cohort, we noticed that the significant different infiltration scores of 3 immune cells (macrophages, mast cells and Treg) and 1 immune-related function (antigen processing cell (APC) co-stimulation) were validated also (Fig. 10c-d).

\section{Discussion}

In the current study, the expression and clinical data were retrieved from the KIRC cohort in the TCGA and the E-MTAB-1980 cohort in the ArrayExpress. Within 60 ferroptosis-related genes, we performed the differential expression analysis and the univariate Cox analysis to screen out 32 prognostic DEGs, from which the Lasso-penalized Cox regression analysis was applied to construct a prognostic 12-gene signature including CARS1, HMGCR, CHAC1, GOT1, CD44, STEAP3, AKR1C1, CBS, DPP4, FANCD2, SLC1A5 and NCOA4. The signature-correlated risk score of each patient in both cohorts was calculated, and according to which, patients were assigned to either high-, or low-risk group divided by the median risk score of the KIRC cohort. Then, the independent prognostic value of the signature was further assessed by K-M survival, ROC and Cox regression analyses in the KIRC cohort, and validated in the EMTAB-1980 cohort. Lastly, risk-related DEGs were identified in both cohorts and applied with the 
enrichment analyses for GO, KEGG and immune infiltration. As expected, several iron-related GO and KEGG terms were significantly enriched. But interestingly, some immune-related terms were identified as well. Further immune infiltration analysis showed that 3 immune cells and 1 immune-related function were enriched in both cohorts which supported the potential relevancy between tumor immune and ferroptosis in ccRCC.

The prognostic 12-ferroptosis-related-gene signature contains 5 protective genes (HMGCR, GOT1, AKR1C1, DPP4 and NCOA4) and 7 risk genes (CARS1, CHAC1, CD44, STEAP3, CBS, FANCD2 and SLC1A5), which can be classified as iron-metabolism-related (NCOA4, STEAP3 and FANCD2), lipidmetabolism-related (HMGCR, AKR1C1 and DPP4), (anti)oxidant-metabolism-related (CHAC1, CD44, CBS and CARS1) and energy-metabolism-related genes (GOT1 and SLC1A5) according to the potential generegulating function for ferroptosis (30).

In iron metabolism, NCOA4 can help elevating the levels of free iron by recruiting iron-storage protein ferritin (FTH) including ferritin light chain (FTL) and ferritin heavy chain 1 (FTH1) for lysosomal degradation and then releasing iron. As a participant of free radical formation and lipid peroxidation propagation, the accumulation of iron can increase the ferroptosis sensitivity of cells. Therefore, inhibition of NCOA4 can suppress ferroptosis induced by amino acid/cystine deprivation or erastin (48, 49). In another way, STEAP3, a metalloreductase reducing $\mathrm{Fe} 3+$ to $\mathrm{Fe} 2+$, can also be up-regulated in ferroptosis. In the endosome, Fe2 + reduced by STEAP3 will be released into the cytosol to increase free iron and therefore participate in ferroptosis. Different from what was mentioned above, FANCD2 is a nuclear protein involved in DNA damage repair with a potential iron down-regulatory function. In bone marrow stromal cells, the knockout of FANCD2 can increase the expression of STEAP3 and enhance erastin-induced ferroptosis (50).

HMGCR is a reductase that can catalyze 3-hydroxy-3-methyl-glutaryl coenzyme A (HMG-CoA) to synthesize mevalonic acid and then take part in the synthesis of sterols coenzyme Q10 (CoQ10), an endogenous suppressor of ferroptosis. A study has shown that the drug inhibition of HMGCR is responsible for the enhancement of FIN56-induced ferroptosis (51). AKR1C1 is a member of aldosterone reductase family 1 (AKR1), an aldehyde detoxification enzymes family that is involved in steroid metabolism. The overexpression of AKR1C (including AKR1C1, AKR1C2 and AKR1C3) has been proved to have an anti-ferroptosis effect through the reduction reaction of converting the end products of lipid peroxides to the corresponding nontoxic lipid-derived alcohols (52). DPP4 is a binding protein to NOX, a participant in a membrane-bound enzyme complex that produce downstream ROS. The combination of NOX-DPP4/CD26 can cause plasma membrane lipid peroxidation and therefore result in ferroptosis, which can be blocked by p53 through DPP4 silencing in colorectal cancer cells (53). In addition, the implication of DPP4 and p53 was also observed in Golgi stress-induced ferroptosis (54).

At the (Anti)oxidant Metabolism in ferroptosis, cysteine serves as an initiator by providing materials for the biosynthesis of glutathione (GSH) which contribute an anti-ferroptosis effect. In one way, extracellular cysteine can be transported into the cytosol by exchanging with intracellular glutamate through the 
cysteine-glutamate exchange system Xc-. CHAC1 and CD44 have been suggested with potential interactions with the system Xc- and providing a pro-ferroptosis effect in Burkitt's Lymphoma (55) and anti-ferroptosis effect in human gastrointestinal cancer (56), respectively. In another way, homocysteine has an alternative transsulfuration pathway that produces cystathionine promoted by CBS and then cysteine promoted by cystathionine (CTH). In the cytoplasm, cysteine can be charged with tRNACys which catalyzed by CARS1 and therefore result in cysteine decreasing. A study has showed that the knockdown of CARS1 can upregulate the compensatory transsulfuration pathway to increase cysteine and suppress ferroptosis induced by erastin, which can be resensitized by silencing CBS (57). Also, the ferroptosis enhancing effect by suppressing CBS has been demonstrated in hepatocellular carcinoma cells (58).

GOT1 and SLC1A5 were both involve in the energy-metabolic network for ROS production in ferroptosis. In cystine deprivation- or erastin-induced ferroptosis, SLC1A5-mediated L-glutamine uptake is a critical process for the production of glutamate, which was further converted into a-ketoglutarate (aKG) by transaminase GOT1-mediated transamination (59). The accumulation of aKG can be converted into acetyl coenzyme A (acetyl-CoA) in the cytoplasm for lipid biosynthesis and fatty acid synthesis or increase mitochondrial ROS and iron level to promote ferroptosis $(60,61)$.

As we all known, immune cells will be drawn and gathered by a set of signals to help programming cell death during apoptosis (62). It is conceivable that similar signal patterns will attract APCs and other immune cells to assist the accomplishment of ferroptosis, although still lacking solid proof. However, an in vitro study about macrophage clearance of ferroptotic cells supported this possibility (63).

Bioinformatically, several studies have demonstrated the potential connection between RCC and immune infiltration $(64,65)$. Clinically, besides palliative targeted therapy, considerable promising results of monotherapy from novel immunotherapy like immune checkpoint inhibitors (ICI) have been observed in some advanced RCC patients (66). Moreover, a combinatory ICl therapy of nivolumab plus ipilimumab has been approved for the phase-3 clinical trial last year (67).

In the presented study, with immune annotation analysis based on risk groups, we discovered that macrophages, mast cells, Treg and an immune-related function APC co-stimulation were commonly enriched in both cohorts with statistical significance, which indicate a potential underlying modulation between tumor immunity and ferroptosis in ccRCC. Macrophages, mast cells and Treg are all APCs that are capable of presenting the processed antigen to $T$ cells and activating the immune response by costimulation. Tumor-associated macrophages (TAMs) play a dual character that can be either pro-cancer or anti-cancer in the immune system $(68,69)$. As in ccRCC, it has been demonstrated that the increased density of TAMs was associated with poor clinical prognosis and aggressive tumor migration $(70,71)$. Similarly, Treg shows a tumor facilitating potential in ccRCC. Treg has been proved to have an association with worse prognosis in $\operatorname{ccRCC}(72,73)$. As for mast cells, the research of Şenbabaoğlu et al. revealed that mast cell density has an independently negative correlation with OS and progression-free survival (PFS) in cCRCC (65). What's more, observing the work of Fu et al., mast cell was negatively correlated with cancer-specific survival (CSS) and relapse-free survival (RFS) in ccRCC independently(74). 
In addition, in vitro and in vivo experiments have demonstrated the angiogenesis promoting effect of mast cells in RCC (75). Although multiple pieces of research have been trying to explore the secrets within macrophages, mast cells and Treg in $\mathrm{CCRCC}$, the underlying mechanism remains poorly characterized, and even more barren in the field of ferroptosis. Relationships between immune response and ferroptosis, and how they correlate to the prognosis in $\mathrm{CCRCC}$ still require further investigation.

Several limitations were observed in the presented study. As a bioinformatics analysis, the weakness of lacking experimental and clinical validation is inevitable, as well as the various possible results from using different cut-off criteria, statistical methods or analyzing tools. Also, establishing a prognostic model by considering a single hallmark might lead to the regrettable missing of many other promising prognostic genes.

In summary, we established a novel ferroptosis-related 12-gene signature which was proved as an independent prognostic predictor for OS in ccRCC. By functional annotation analyses, the gene signature was inferred as tumor-immunity-correlated, however, the underlying mechanism is still poorly characterized and needed further exploration.

\section{Declarations}

Ethics approval and consent to participate: Not applicable.

Consent for publication: Not applicable.

Availability of data and materials: The cohorts analyzed for this study can be found in the TCGA database (www.cancergenome.nih.gov) and ArrayExpress database (https://www.ebi.ac.uk/arrayexpress).

Competing interests: The authors declare that they have no competing interests.

Funding: This work was supported by the Guangdong Medical Research Foundation (A2018103 and 201711795717822), Shantou Science and Technology Project (180404094011031) and Natural Science Foundation of Guangdong Province (No. 2015A030310078).

Authors' contributions: Conceptualization, Peilin Shen; Data curation, Mingen Lin, Dehua Ou and Zhuangkai Huang; Formal analysis, Yingkai Hong and Dehua Ou; Methodology, Peilin Shen; Project administration, Peilin Shen; Software, Yingkai Hong and Mingen Lin; Visualization, Yingkai Hong and Mingen Lin; Writing - original draft, Yingkai Hong; Writing - review \& editing, Peilin Shen. All authors read and approved the final manuscript.

Acknowledgements: Not applicable.

\section{References}


1. Wild CP WE, Stewart BW. World Cancer Report: Cancer Research for Cancer Prevention Lyon, France: International Agency for Research on Cancer; 2020.

2. Prasad SR, Humphrey PA, Catena JR, Narra VR, Srigley JR, Cortez AD, et al. Common and Uncommon Histologic Subtypes of Renal Cell Carcinoma: Imaging Spectrum with Pathologic Correlation. Radiographics. 2006;26(6):1795-806.

3. Rao A, Wiggins C, Lauer RC. Survival outcomes for advanced kidney cancer patients in the era of targeted therapies. Case Rep Rheumatol. 2018;6(9):165-

4. Gill DM, Agarwal N, Vaishampayan U. Evolving Treatment Paradigm in Metastatic Renal Cell Carcinoma. American Society of Clinical Oncology educational book American Society of Clinical Oncology Annual Meeting. 2017;37:319-29.

5. Manz DH, Blanchette NL, Paul BT, Torti FM, Torti SV. Iron and cancer: recent insights. Annals of the New York Academy of Sciences. 2016;1368(1).

6. Huang X. Iron overload and its association with cancer risk in humans: evidence for iron as a carcinogenic metal. Mutation Research. 2003;533(1-2):153-71.

7. Weinlich R, Oberst A, Beere HM, Green DR. Necroptosis in development, inflammation and disease. Nat Rev Mol Cell Biol. 2016;18(2):127.

8. Bergsbaken T, Fink SL, Cookson BT. Pyroptosis: host cell death and inflammation. Nature reviews Microbiology. 2009;7(2):99-109.

9. Song X, Zhu S, Xie Y, Liu J, Sun L, Zeng D, et al. JTC801 Induces pH-dependent Death Specifically in Cancer Cells and Slows Growth of Tumors in Mice. Gastroenterology. 2018;154(5):1480-93.

10. Liu J, Kuang F, Kang R, Tang D. Alkaliptosis: a new weapon for cancer therapy. Cancer Gene Therapy. 2020.

11. Dixon SJ, Lemberg KM, Lamprecht MR, Skouta R, Zaitsev EM, Gleason CE, et al. Ferroptosis: an irondependent form of nonapoptotic cell death. Cell. 2012;149(5):1060-72.

12. Chen X, Li J, Kang R, Klionsky DJ, Tang D. Ferroptosis: machinery and regulation. Autophagy. 2020:128.

13. Tang D, Chen X, Kang R, Kroemer G. Ferroptosis: molecular mechanisms and health implications. Cell research. 2020.

14. Yang WS, Sriramaratnam R, Welsch ME, Shimada K, Skouta R, Viswanathan VS, et al. Regulation of Ferroptotic Cancer Cell Death by GPX4. Cell. 2014;156(1-2):317-31.

15. Vasuri F, Visani M, Acquaviva G, Brand T, Fiorentino M, Pession A, et al. Role of microRNAs in the main molecular pathways of hepatocellular carcinoma. World Journal of Gastroenterology. 2018;24(25):2647-60.

16. Hao S, Yu J, He W, Huang Q, Zhao Y, Liang B, et al. Cysteine Dioxygenase 1 Mediates Erastin-Induced Ferroptosis in Human Gastric Cancer Cells. Neoplasia. 2017;19(12):1022-32.

17. Ishimoto T, Nagano O, Yae T, Tamada M, Motohara T, Oshima H, et al. CD44 variant regulates redox status in cancer cells by stabilizing the XCT subunit of system xc(-) and thereby promotes tumor 
growth. Cancer Cell. 2011;19(3):387-400.

18. Basuli D, Tesfay L, Deng Z, Paul B, Torti SV. Iron addiction: A novel therapeutic target in ovarian cancer. Oncogene. 2017;36(29).

19. Greenshields AL, Shepherd TG, Hoskin DW. Contribution of reactive oxygen species to ovarian cancer cell growth arrest and killing by the anti-malarial drug artesunate. Molecular Carcinogenesis. 2017.

20. Ma S, Dielschneider RF, Henson ES, Xiao W, Gibson SB. Ferroptosis and autophagy induced cell death occur independently after siramesine and lapatinib treatment in breast cancer cells. Plos One. 2017;12(8):e0182921.

21. Zhu HY, Huang ZX, Chen GQ, Sheng F, Zheng YS. Typhaneoside prevents acute myeloid leukemia (AML) through suppressing proliferation and inducing ferroptosis associated with autophagy. Biochemical and biophysical research communications. 2019;516(4):1265-71.

22. Xie Y, Hou W, Song X, Yu Y, Huang J, Sun X, et al. Ferroptosis: process and function. Cell Death \& Differentiation. 2016;23(3):369-79.

23. Haase VH. Hypoxic regulation of erythropoiesis and iron metabolism. American journal of physiology Renal physiology. 2010;299(1):F1-13.

24. Zhang S, Chang W, Wu H, Wang YH, Wang ZP. Pan-cancer analysis of iron metabolic landscape across the Cancer Genome Atlas. Journal of Cellular Physiology. 2020(5).

25. Moon D, Kim J, Yoon SP. Yeast extract inhibits the proliferation of renal cell carcinoma cells via regulation of iron metabolism. Molecular Medicine Reports. 2019;20(4):3933-41.

26. Team RC. R: A language and environment for statistical computing. R Foundation for Statistical Computing, Vienna, Austria. 2020 [Available from: https://www.R-project.org/.

27. Stockwell BR, Friedmann Angeli JP, Bayir H, Bush Al, Conrad M, Dixon SJ, et al. Ferroptosis: A Regulated Cell Death Nexus Linking Metabolism, Redox Biology, and Disease. Cell. 2017;171(2):27385.

28. Bersuker K, Hendricks JM, Li Z, Magtanong L, Ford B, Tang PH, et al. The CoQ oxidoreductase FSP1 acts parallel to GPX4 to inhibit ferroptosis. Nature. 2019;575(7784):688-92.

29. Doll S, Freitas FP, Shah R, Aldrovandi M, da Silva MC, Ingold I, et al. FSP1 is a glutathioneindependent ferroptosis suppressor. Nature. 2019;575(7784):693-8.

30. Hassannia B, Vandenabeele P, Vanden Berghe T. Targeting Ferroptosis to Iron Out Cancer. Cancer Cell. 2019;35(6):830-49.

31. Robinson MD, McCarthy DJ, Smyth GK. edgeR: a Bioconductor package for differential expression analysis of digital gene expression data. Bioinformatics (Oxford, England). 2010;26(1):139-40.

32. Kolde R. pheatmap: Pretty Heatmaps. R package version 1.0.12. 2019 [Available from: https://CRAN.R-project.org/package=pheatmap.

33. Dusa A. venn: Draw Venn Diagrams. R package version 1.9. 2020 [Available from: https://CRAN.Rproject.org/package=venn. 
34. Szklarczyk D, Gable AL, Lyon D, Junge A, Wyder S, Huerta-Cepas J, et al. STRING v11: protein-protein association networks with increased coverage, supporting functional discovery in genome-wide experimental datasets. Nucleic acids research. 2019;47(D1):D607-d13.

35. Csardi G, Nepusz T. The Igraph Software Package for Complex Network Research. InterJournal. 2005;Complex Systems:1695.

36. Wickham H. Reshaping Data with the reshape Package. Journal of statistical software. 2007;21:120.

37. Simon N, Friedman J, Hastie T, Tibshirani R. Regularization Paths for Cox's Proportional Hazards Model via Coordinate Descent. Journal of statistical software. 2011;39(5):1-13.

38. Tibshirani R. The lasso method for variable selection in the Cox model. Statistics in medicine. 1997;16(4):385-95.

39. Friedman J, Hastie T, Tibshirani R. Regularization Paths for Generalized Linear Models via Coordinate Descent. Journal of statistical software. 2010;33(1):1-22.

40. Krijthe JH. Rtsne: T-Distributed Stochastic Neighbor Embedding using a Barnes-Hut Implementation 2015 [Available from: https://github.com/jkrijthe/Rtsne.

41. T T. A Package for Survival Analysis in R. R package version 3.2-7 2020 [Available from: https://CRAN.R-project.org/package=survival.

42. Ritchie ME, Phipson B, Wu D, Hu Y, Law CW, Shi W, et al. limma powers differential expression analyses for RNA-sequencing and microarray studies. Nucleic acids research. 2015;43(7):e47.

43. Kanehisa M, Goto S. KEGG: kyoto encyclopedia of genes and genomes. Nucleic acids research. 2000;28(1):27-30.

44. Ogata H, Goto S, Sato K, Fujibuchi W, Bono H, Kanehisa M. KEGG: Kyoto Encyclopedia of Genes and Genomes. Nucleic acids research. 1999;27(1):29-34.

45. Yu G, Wang LG, Han Y, He QY. clusterProfiler: an R package for comparing biological themes among gene clusters. Omics : a journal of integrative biology. 2012;16(5):284-7.

46. Rooney MS, Shukla SA, Wu CJ, Getz G, Hacohen N. Molecular and genetic properties of tumors associated with local immune cytolytic activity. Cell. 2015;160(1-2):48-61.

47. Hänzelmann S, Castelo R, Guinney J. GSVA: gene set variation analysis for microarray and RNA-seq data. BMC bioinformatics. 2013;14:7.

48. Gao M, Monian P, Pan Q, Zhang W, Xiang J, Jiang X. Ferroptosis is an autophagic cell death process. Cell research. 2016;26(9):1021-32.

49. Hou W, Xie Y, Song X, Sun X, Lotze MT, Zeh HJ, 3rd, et al. Autophagy promotes ferroptosis by degradation of ferritin. Autophagy. 2016;12(8):1425-8.

50. Song X, Xie Y, Kang R, Hou W, Sun X, Epperly MW, et al. FANCD2 protects against bone marrow injury from ferroptosis. Biochemical and biophysical research communications. 2016;480(3):443-9.

51. Shimada K, Skouta R, Kaplan A, Yang WS, Hayano M, Dixon SJ, et al. Global survey of cell death mechanisms reveals metabolic regulation of ferroptosis. Nature chemical biology. 2016;12(7):497- 
503.

52. Dixon SJ, Patel DN, Welsch M, Skouta R, Lee ED, Hayano M, et al. Pharmacological inhibition of cystine-glutamate exchange induces endoplasmic reticulum stress and ferroptosis. eLife. 2014;3:e02523.

53. Xie Y, Zhu S, Song X, Sun X, Fan Y, Liu J, et al. The Tumor Suppressor p53 Limits Ferroptosis by Blocking DPP4 Activity. Cell reports. 2017;20(7):1692-704.

54. Alborzinia H, Ignashkova TI, Dejure FR, Gendarme M, Theobald J, Wölfl S, et al. Golgi stress mediates redox imbalance and ferroptosis in human cells. Communications biology. 2018;1:210.

55. Wang N, Zeng GZ, Yin JL, Bian ZX. Artesunate activates the ATF4-CHOP-CHAC1 pathway and affects ferroptosis in Burkitt's Lymphoma. Biochemical and biophysical research communications. 2019;519(3):533-9.

56. Ishimoto T, Nagano O, Yae T, Tamada M, Motohara T, Oshima H, et al. CD44 variant regulates redox status in cancer cells by stabilizing the xCT subunit of system xc(-) and thereby promotes tumor growth. Cancer Cell. 2011;19(3):387-400.

57. Hayano M, Yang WS, Corn CK, Pagano NC, Stockwell BR. Loss of cysteinyl-tRNA synthetase (CARS) induces the transsulfuration pathway and inhibits ferroptosis induced by cystine deprivation. Cell death and differentiation. 2016;23(2):270-8.

58. Wang L, Cai H, Hu Y, Liu F, Huang S, Zhou Y, et al. A pharmacological probe identifies cystathionine $\beta$ synthase as a new negative regulator for ferroptosis. Cell death \& disease. 2018;9(10):1005.

59. Gao M, Monian P, Quadri N, Ramasamy R, Jiang X. Glutaminolysis and Transferrin Regulate Ferroptosis. Molecular cell. 2015;59(2):298-308.

60. Lee H, Zandkarimi F, Zhang Y, Meena JK, Kim J, Zhuang L, et al. Energy-stress-mediated AMPK activation inhibits ferroptosis. Nature cell biology. 2020;22(2):225-34.

61. Shin D, Lee J, You JH, Kim D, Roh JL. Dihydrolipoamide dehydrogenase regulates cystine deprivationinduced ferroptosis in head and neck cancer. Redox biology. 2020;30:101418.

62. Elliott MR, Ravichandran KS. The Dynamics of Apoptotic Cell Clearance. Developmental cell. 2016;38(2):147-60.

63. Klöditz K, Fadeel B. Three cell deaths and a funeral: macrophage clearance of cells undergoing distinct modes of cell death. Cell death discovery. 2019;5:65.

64. Zhang S, Zhang E, Long J, Hu Z, Peng J, Liu L, et al. Immune infiltration in renal cell carcinoma. Cancer science. 2019;110(5):1564-72.

65. Şenbabaoğlu Y, Gejman RS, Winer AG, Liu M, Van Allen EM, de Velasco G, et al. Tumor immune microenvironment characterization in clear cell renal cell carcinoma identifies prognostic and immunotherapeutically relevant messenger RNA signatures. Genome biology. 2016;17(1):231.

66. Joseph RW, Chatta G, Vaishampayan U. Nivolumab treatment for advanced renal cell carcinoma: Considerations for clinical practice. Urologic oncology. 2017;35(4):142-8. 
67. Cella D, Grünwald V, Escudier B, Hammers HJ, George S, Nathan P, et al. Patient-reported outcomes of patients with advanced renal cell carcinoma treated with nivolumab plus ipilimumab versus sunitinib (CheckMate 214): a randomised, phase 3 trial. The Lancet Oncology. 2019;20(2):297-310.

68. Kitamura T, Qian BZ, Pollard JW. Immune cell promotion of metastasis. Nature reviews Immunology. 2015;15(2):73-86.

69. Sica A, Larghi P, Mancino A, Rubino L, Porta C, Totaro MG, et al. Macrophage polarization in tumour progression. Seminars in cancer biology. 2008;18(5):349-55.

70. Komohara Y, Hasita H, Ohnishi K, Fujiwara Y, Suzu S, Eto M, et al. Macrophage infiltration and its prognostic relevance in clear cell renal cell carcinoma. Cancer science. 2011;102(7):1424-31.

71. Kadomoto S, Izumi K, Hiratsuka K, Nakano T, Naito R, Makino T, et al. Tumor-Associated Macrophages Induce Migration of Renal Cell Carcinoma Cells via Activation of the CCL20-CCR6 Axis. Cancers. 2019;12(1).

72. Nakayama T, Saito K, Kumagai J, Nakajima Y, Kijima T, Yoshida S, et al. Higher Serum C-reactive Protein Level Represents the Immunosuppressive Tumor Microenvironment in Patients With Clear Cell Renal Cell Carcinoma. Clinical genitourinary cancer. 2018;16(6):e1151-e8.

73. Li JF, Chu YW, Wang GM, Zhu TY, Rong RM, Hou J, et al. The prognostic value of peritumoral regulatory $T$ cells and its correlation with intratumoral cyclooxygenase-2 expression in clear cell renal cell carcinoma. BJU international. 2009;103(3):399-405.

74. Fu H, Zhu Y, Wang Y, Liu Z, Zhang J, Wang Z, et al. Tumor Infiltrating Mast Cells (TIMs) Confers a Marked Survival Advantage in Nonmetastatic Clear-Cell Renal Cell Carcinoma. Annals of surgical oncology. 2017;24(5):1435-42.

75. Chen Y, Li C, Xie H, Fan Y, Yang Z, Ma J, et al. Infiltrating mast cells promote renal cell carcinoma angiogenesis by modulating $\mathrm{PI} 3 \mathrm{AK} \rightarrow \mathrm{AKT} \rightarrow \mathrm{GSK} 3 \beta \rightarrow \mathrm{AM}$ signaling. Oncogene. 2017;36(20):2879-88.

\section{Figures}


Excluded 4 tumor samples:

follow-up with 0 day

\section{Derivation cohort}

KIRC from TCGA

(Tumor samples: 530 Normal samples: 72)

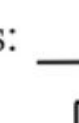

mRNA expression data

Normal samples: 72 Tumor samples: 526

clinical information

Tumor samples: 526

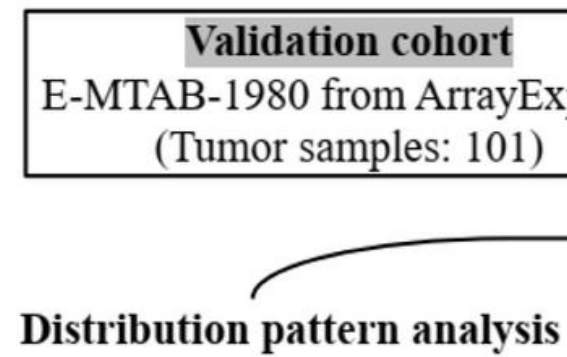

Scatter diagram

PCA t-SNE

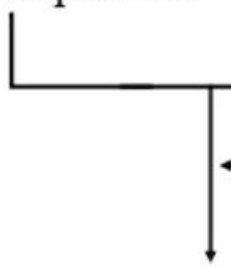

Tumor VS. normal

differential analysis

Ferroptosis-related genes

$(\mathrm{n}=60)$

Univariate Cox analysis of

overall survival

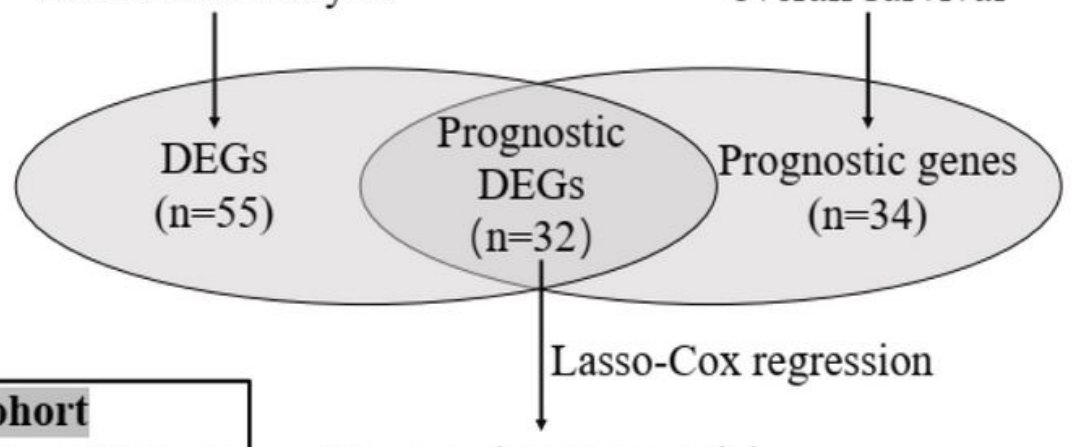

Lasso-Cox regression

\section{2-gene signature model}

Prognostic analysis

K-M Surivival analysis

time-dependent ROC analysis

Cox regression analysis for clinical independence
Functional analysis

GO

KEGG

\section{Figure 1}

Flow chart of bioinformatics analysis. Legends: DEGs, differentially expressed genes; PCA, prin-cipal component analysis; t-SNE, t-distributed stochastic neighbor embedding; K-M, Kaplan-Meier; ROC, receiver operating characteristic; GO, Gene Ontology; KEGG, Kyoto Ency-clopedia of Genes and Genomes; ssGSEA, single-sample gene set enrichment analysis. 


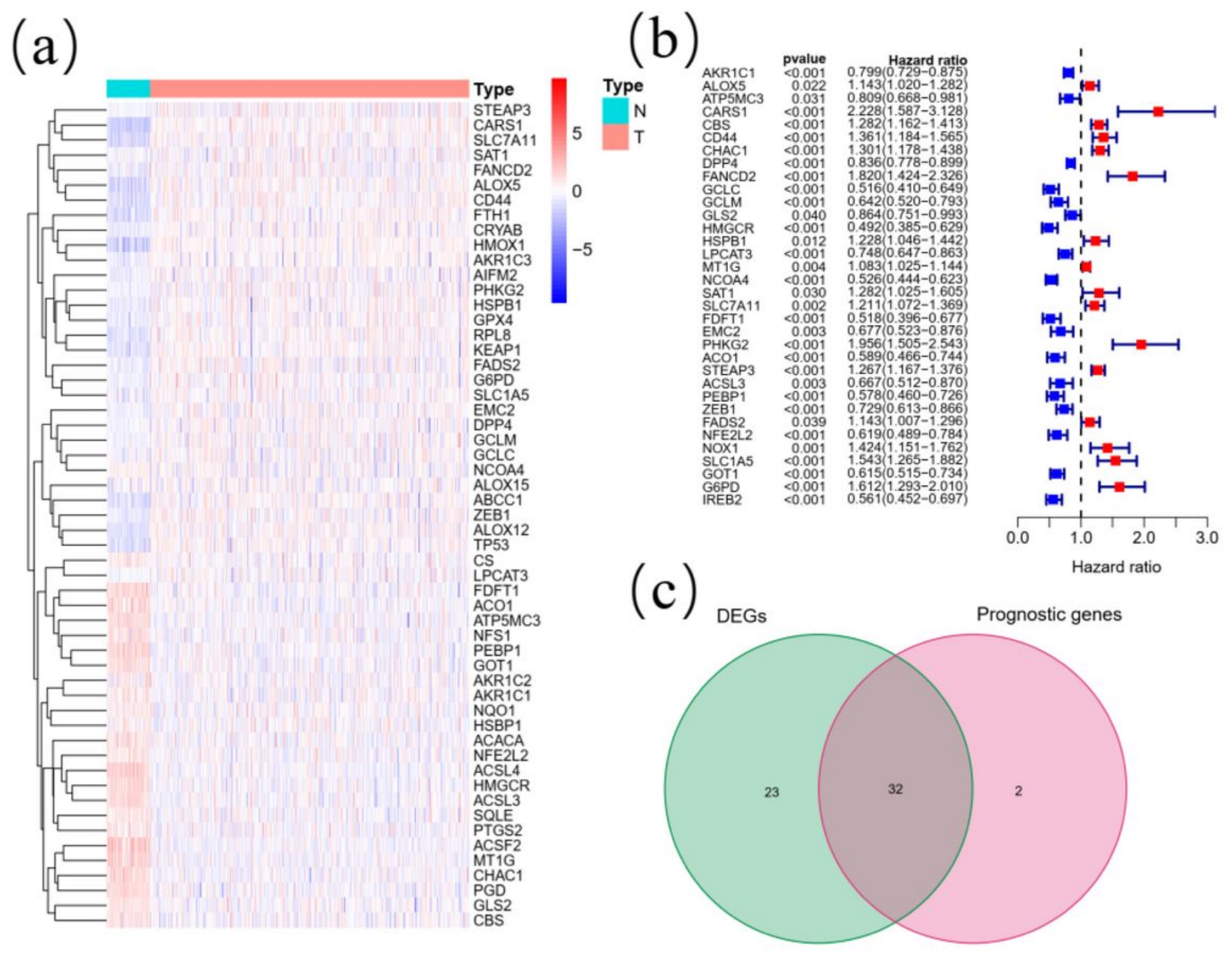

Figure 2

Identification of the prognostic ferroptosis-related DEGs in the KIRC cohort. Legends: (a) Heatmap showing the ferroptosis-related DEGs identified with differential expression analysis. (b) Forest plots showing the significantly prognostic ferroptosis-related genes identified with the univariate Cox regression analysis based on OS. (c) Venn diagram showing the overlapping genes between ferroptosisrelated DEGs and OS-correlated genes. 

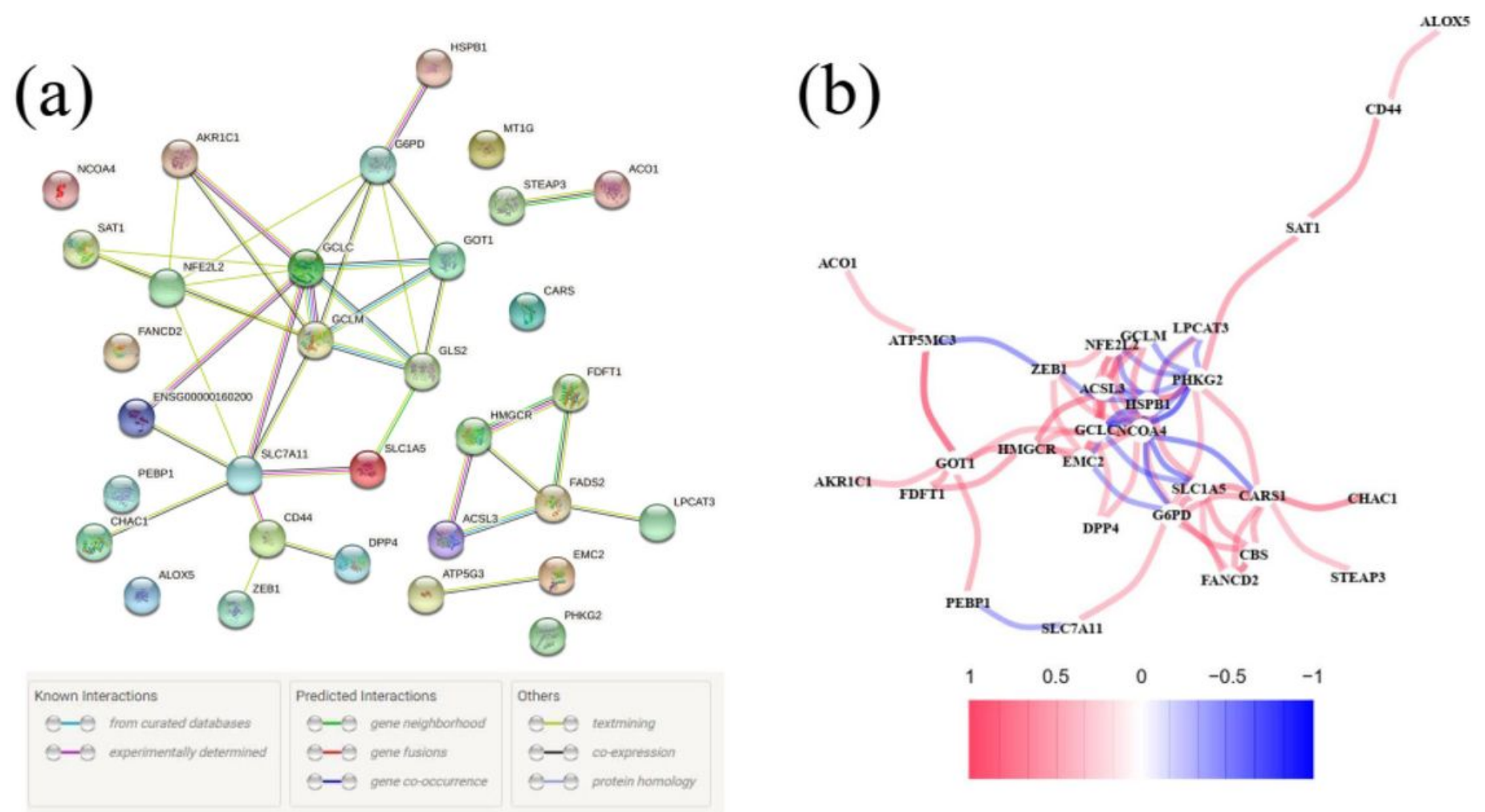

\section{Figure 3}

Interaction networks of the prognostic ferroptosis-related DEGs in the KIRC cohort. Legends: (a) The PPI network downloaded from the STRING database indicated the interactions among the candidate genes. (b) The correlation network of candidate genes, in which the correlation coeffi-cients are represented by different colors. 

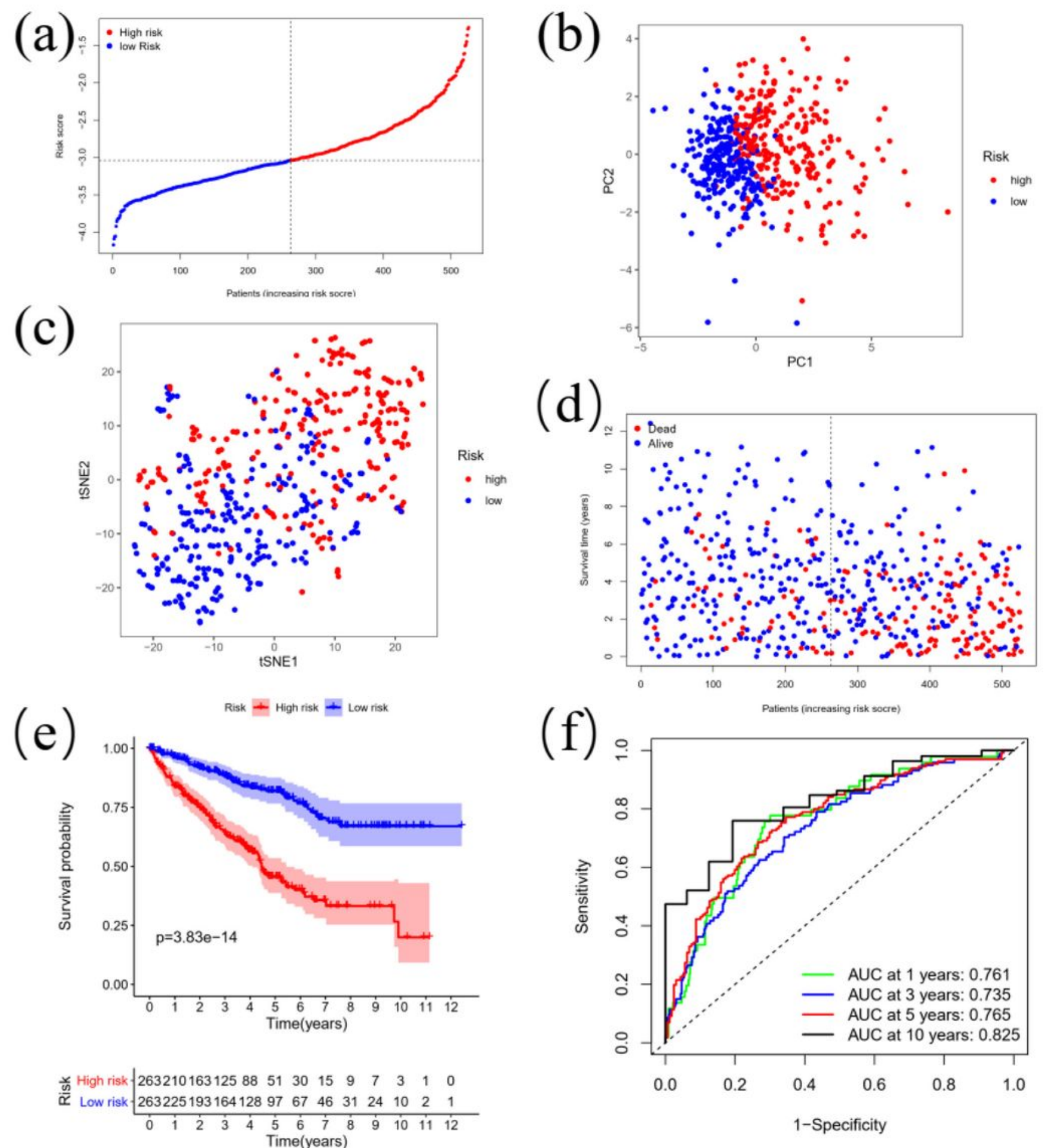

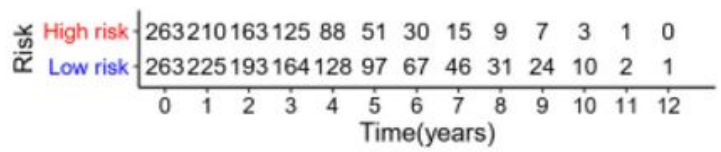

Figure 4

Distribution and prognostic analyses of the 12-gene signature in the KIRC cohort. Legends: (a) The distribution and median value of the risk scores in the KIRC cohort. (b) PCA plot of the KIRC cohort showing the distribution of the established gene signature expression in different risk groups. (c) t-SNE plot of the KIRC cohort showing the distribution of the patients in differ-ent risk groups. (d) The distributions of the risk scores and corresponding survival times of all patients in the KIRC cohort. (e) OS- 
based K-M survival curves for the patients in the high-risk group and low-risk group in the KIRC cohort. (f) AUC of time-dependent ROC curves verified the prognostic performance of the risk score in the KIRC cohort.
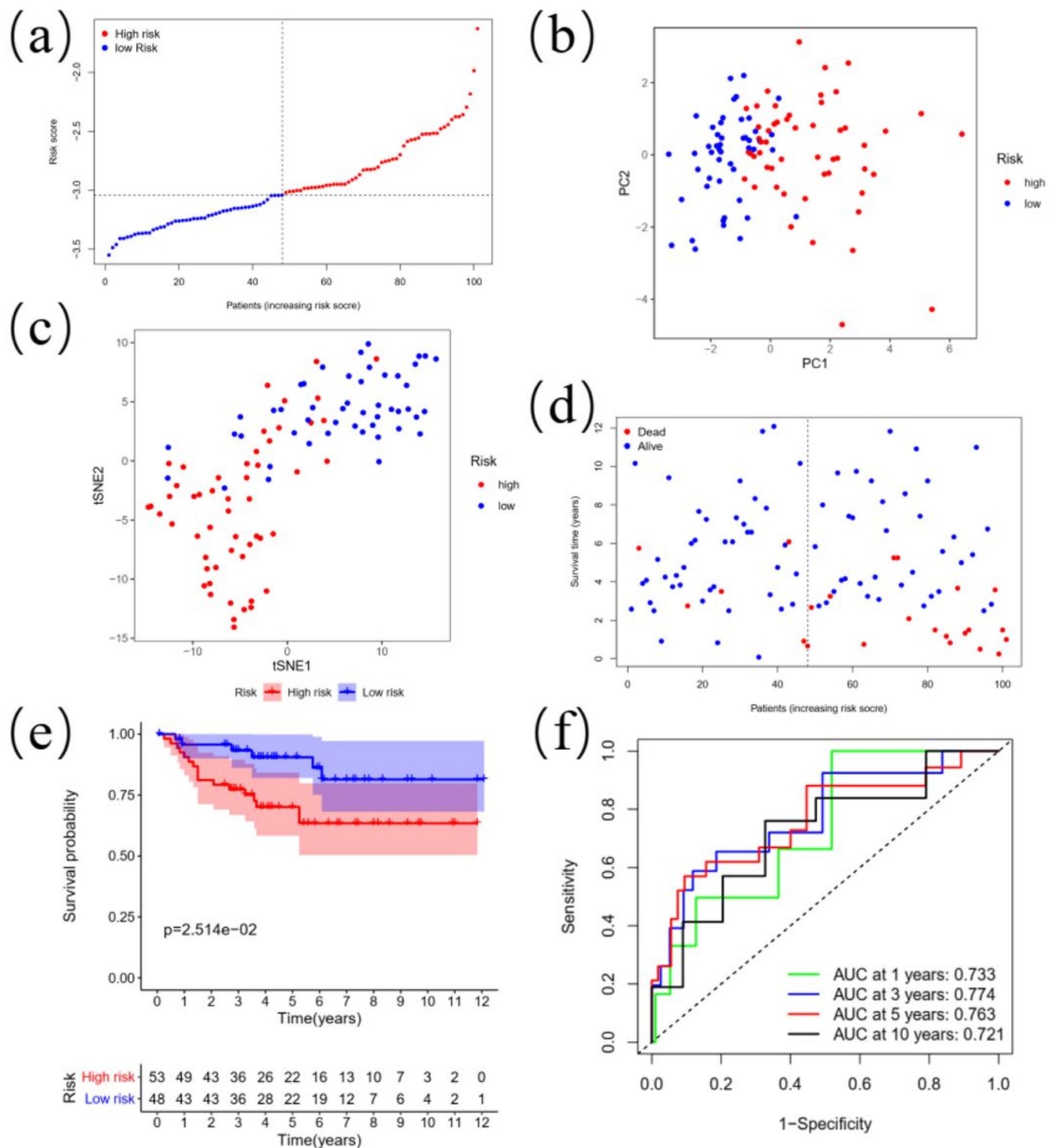

Figure 5

Distribution and prognostic analyses of the 12-gene signature in the E-MTAB-1980 co-hort. Legends: (a) The distribution and median value of the risk scores in the E-MTAB-1980 cohort. (b) PCA plot of the E- 
MTAB-1980 cohort showing the distribution of the established gene signature ex-pression in different risk groups. (c) t-SNE plot of the E-MTAB-1980 cohort showing the distribu-tion of the patients in different risk groups. (d) The distributions of the risk scores and corre-sponding survival times of all patients in the EMTAB-1980 cohort. (e) OS-based K-M survival curves for the patients in the high-risk group and low-risk group in the E-MTAB-1980 cohort. (f) AUC of time-dependent ROC curves verified the prognostic performance of the risk score in the E-MTAB-1980 cohort.

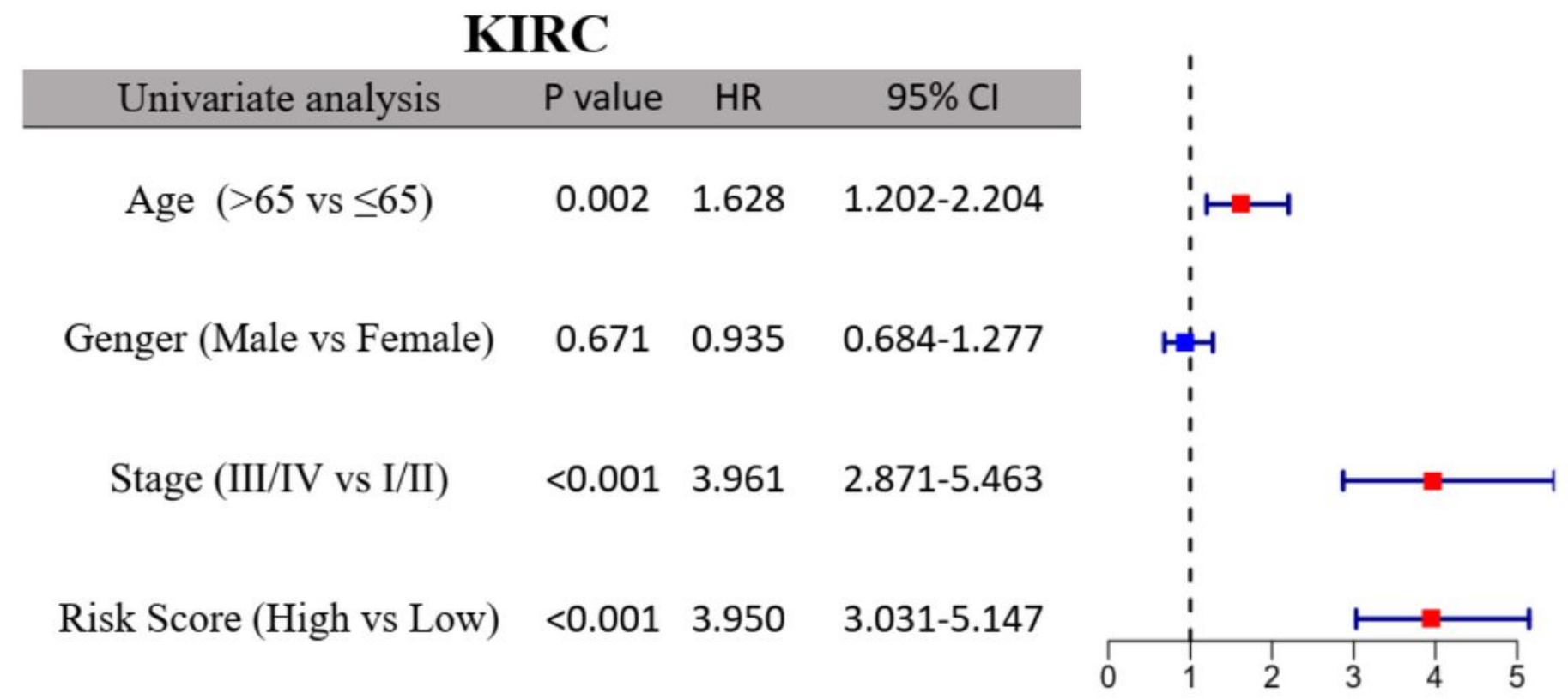

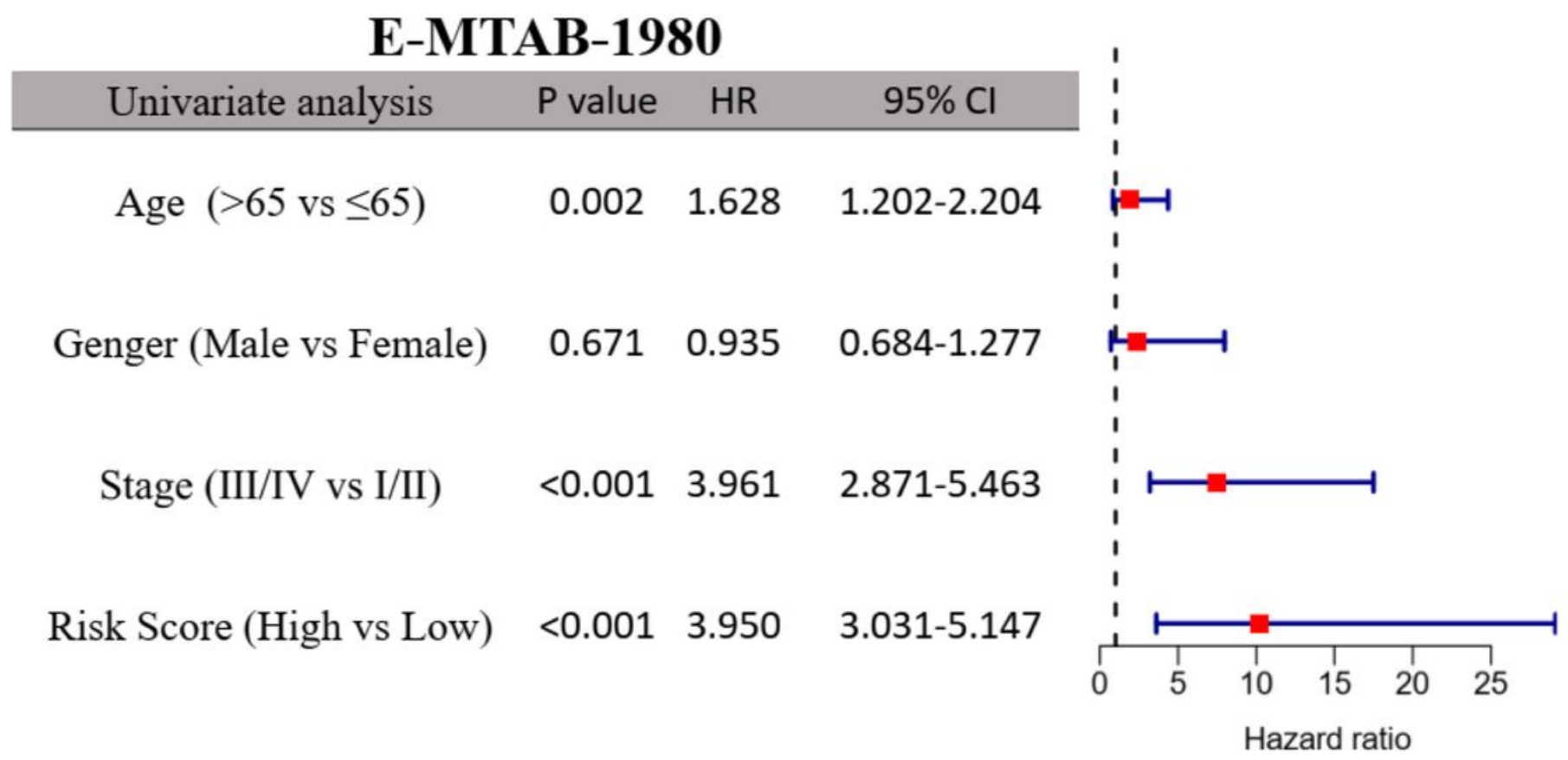

\section{Figure 6}

Univariate Cox regression analysis regarding OS in the KIRC cohort and the E-MTAB-1980 cohort. 
KIRC

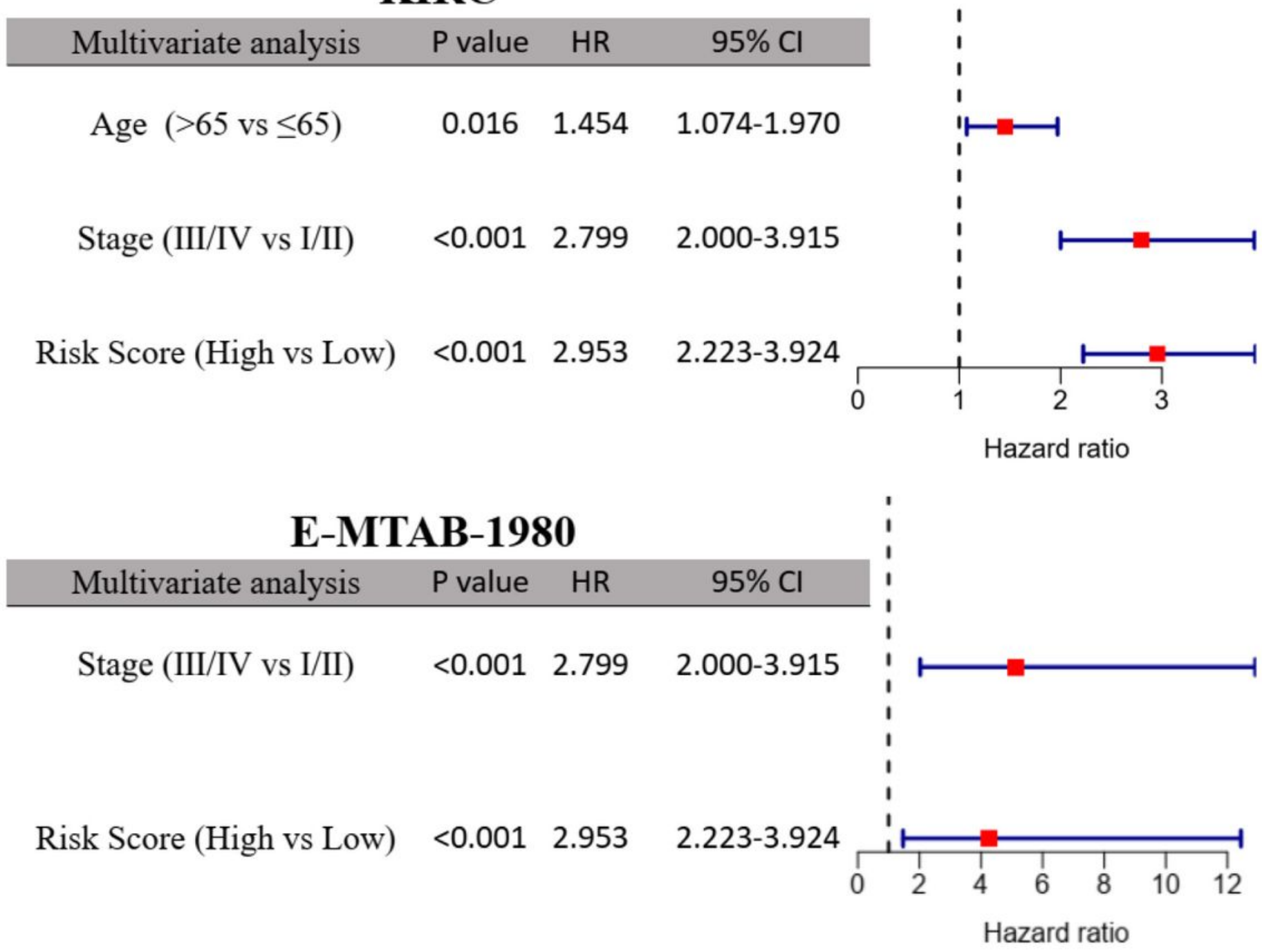

Figure 7

Multivariate Cox regression analysis regarding OS in the KIRC cohort and the E-MTAB-1980 cohort. 


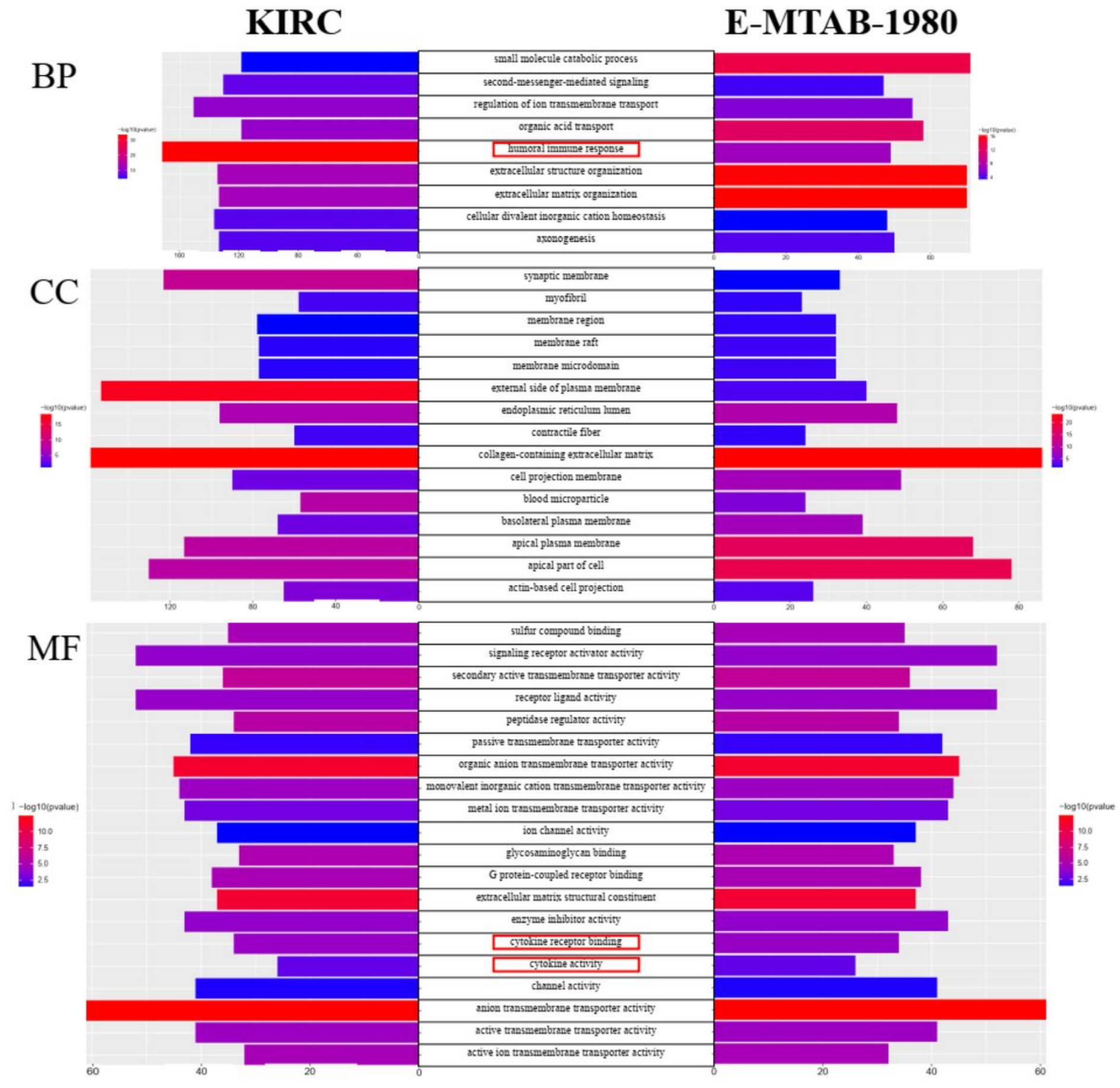

\section{Figure 8}

Results of GO enrichment analysis. Legends: The 9, 15 and 20 overlapping terms of the top 30 en-riched $\mathrm{BP}, \mathrm{CC}$ and MF terms between the KIRC and the E-MTAB-1980 cohort are displayed, re-spectively. The red rectangles indicate the immune-related terms. 


\section{KIRC}

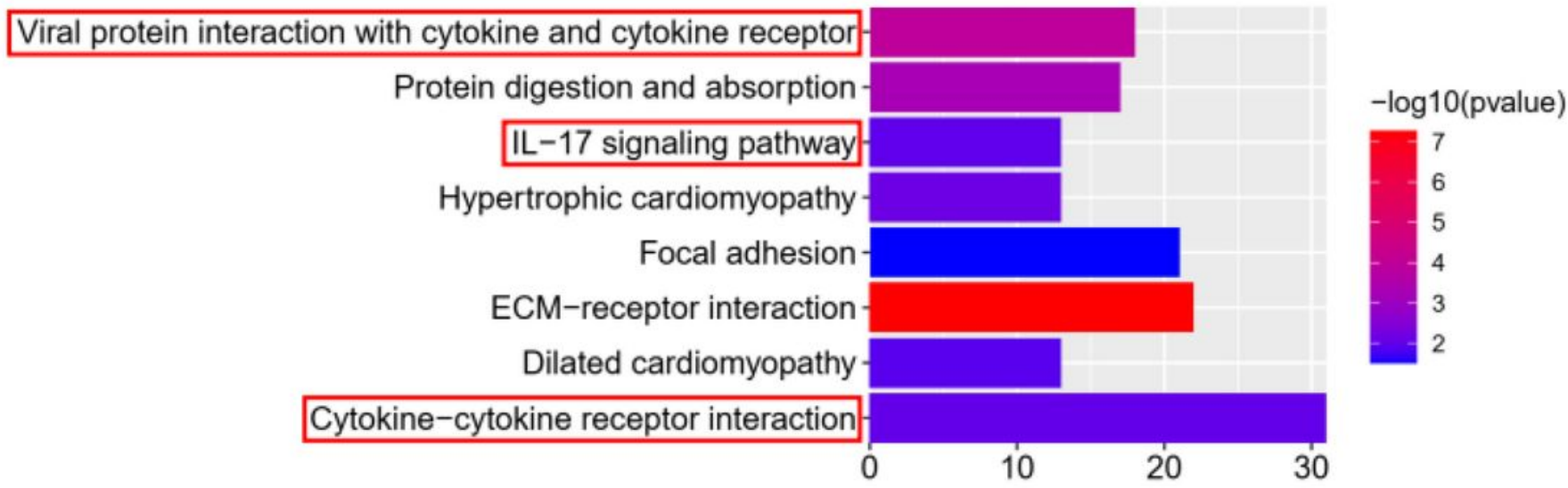

\section{E-MTAB-1980}

Viral protein interaction with cytokine and cytokine receptor

Protein digestion and absorption

IL-17 signaling pathway

Hypertrophic cardiomyopathy

Focal adhesion

ECM-receptor interaction

Dilated cardiomyopathy

Cytokine-cytokine receptor interaction

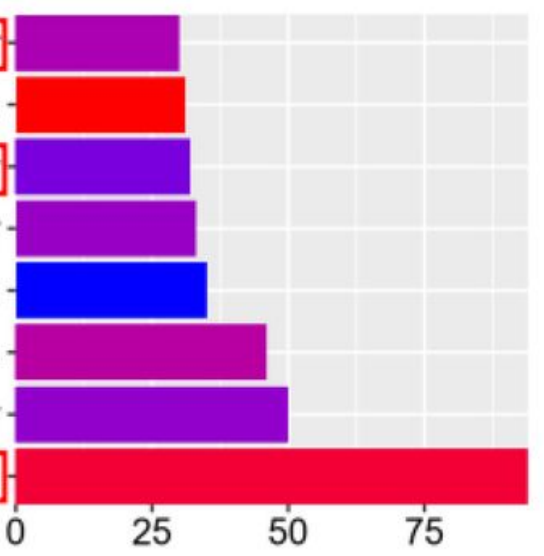

$-\log 10$ (pvalue)

\section{Figure 9}

Results of KEGG enrichment analysis. Legends: The 8 overlapping terms of the top 30 enriched KEGG terms between the KIRC and the E-MTAB-1980 cohort are displayed. The red rectangles indicate the immune-related terms. 
KIRC
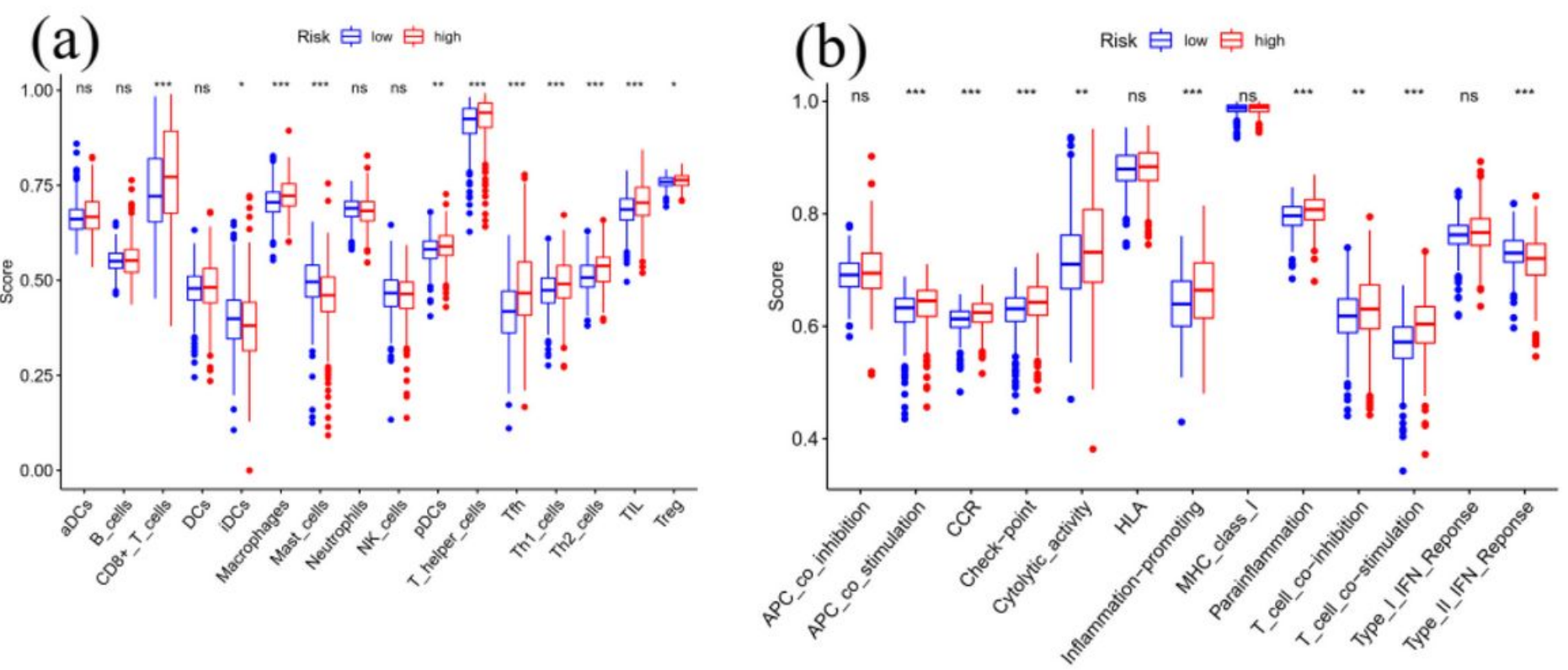

\section{E-MTAB-1980}
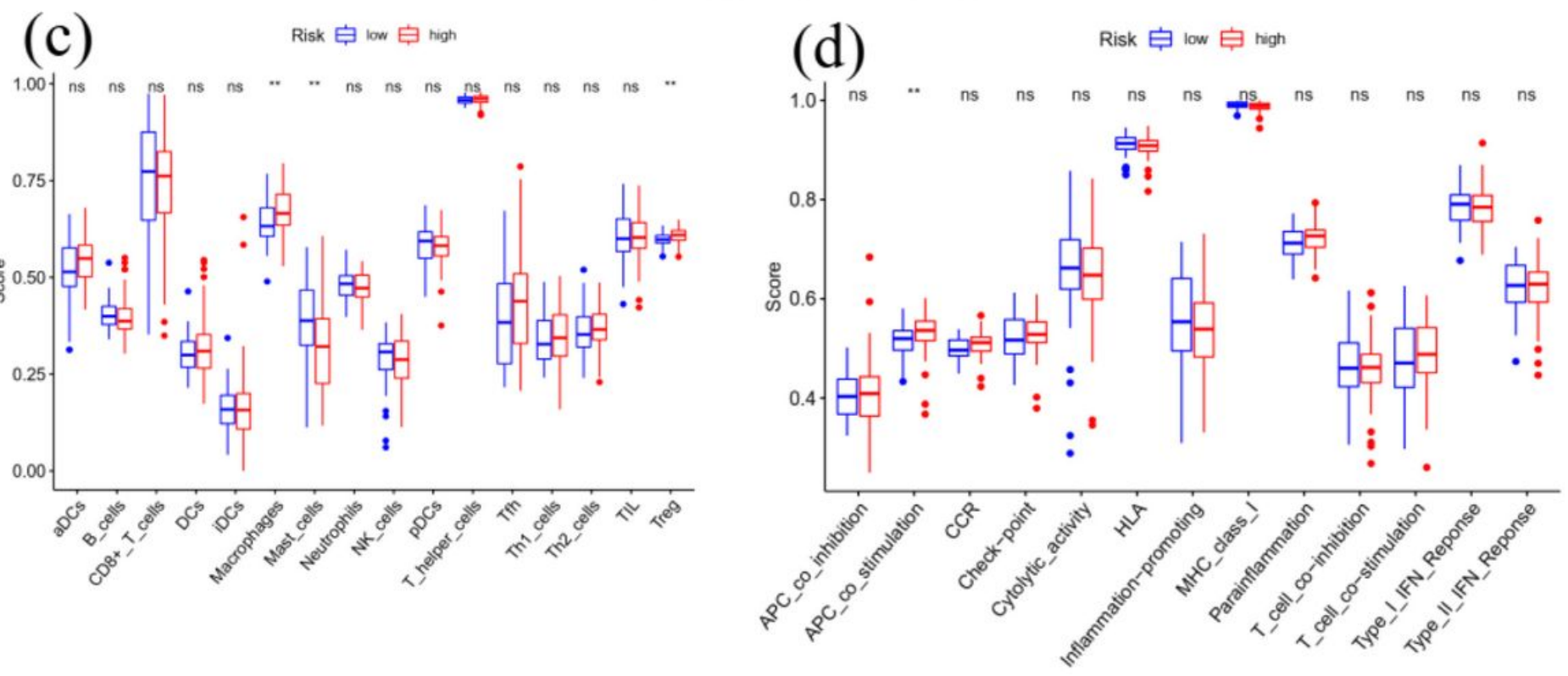

Figure 10

Results of ssGSEA immune infiltration in the KIRC and the E-MTAB-1980 cohort. Legends: (a) The sSGSEA scores of 16 immune cells between different risk groups in the KIRC cohort. (b) The ssGSEA scores of 13 immune-related functions between different risk groups in the KIRC cohort. (c) The ssGSEA scores of 16 immune cells between different risk groups in the E-MTAB-1980 co-hort. (d) The ssGSEA scores of 13 immune-related functions between different risk groups in the E-MTAB-1980 cohort. aDCs, activated dendritic cells; DCs, dendritic cells; iDCs, immature den-dritic cells; NK cells, natural killer cells; 
pDCs, plasmacytoid dendritic cells; Tfh, follicular helper T cell; Th1 cells, helper T cells 1 ; Th2 cells, helper

T cells 2; TIL, tumor infiltrating lymphocyte; Treg, regulatory T cell; CCR, cytokine-cytokine receptor; HLA, human leukocyte antigen; MHC, major histocompatibility complex; IFN, immune interferon; ns, not significant; *, adjusted $\mathrm{P}<0.05$; **, adjusted $\mathrm{P}<0.01$; ***, adjusted $\mathrm{P}<0.001$.

\section{Supplementary Files}

This is a list of supplementary files associated with this preprint. Click to download.

- TableS160ferroptosisrelatedgenes.xlsx

- TableS2AnnotatedgenesetsforssGSEA.xIsx

- TableS3GOandKEGGenrichmentanalysisinKIRC.xlsx

- TableS4GOandKEGGenrichmentanalysisinEMTAB1980.xlsx 